\title{
Peculiarities and populations in elliptical galaxies
}

\section{Dating the last star formation event}

\begin{abstract}
R. Michard
Observatoire de Paris, LERMA, 77 Av. Denfert-Rochereau, 75015 Paris, France

e-mail: raymond.michard@obspm.fr

Received 26 July 2005 / Accepted 17 November 2005

\section{ABSTRACT}

Using 6 colours and 4 Lick line-indices we derive two-component models of the populations of ellipticals, involving a "primary" and a "juvenile" population. The first component is defined by the regressions of indices against the central velocity dispersion found in Papers I and II for the Nop sample of non-peculiar objects. The second one is approximated by an SSP, and the modeling derives its age $A$, metallicity $Z$ and fractional $V$-luminosity $q_{V}$, the fractional mass $q_{M}$ being found therefrom. The model is designed for "blueish" peculiar galaxies, i.e. the Pec sample and NGC 2865 family in the terminology of Paper I. The morphological peculiarities and the population anomaly are then believed to involve the same event, i.e. a merger plus starburst. It is possible to improve the models in a few cases by introducing diffuse dust (as suggested by far IR data), and/or by taking into account the fact that Lick- and colour indices do not relate to identical galaxy volumes. In most of the cases, the mass ratio of young stars $q_{M}$ seems too small for the product of a recent major merger: the events under consideration might be minor mergers bringing "the final touch" to the build-up of the structure of the E-type object. The same modeling has been successfully applied to blueish galaxies of the Nop sample, without morphological peculiarities however, to support the occurence of a distinct perturbing event. A few reddish objects of the Pec sample (NGC 3923 family) and of the Nop sample are also modeled, in terms of an excess of high metallicity stars, or diffuse dust, or both, but the results are inconclusive.
\end{abstract}

Key words. galaxies: elliptical and lenticulars, CD

\section{Introduction}

In Paper I of this series (Michard \& Prugniel 2004) the classical question of the morphological peculiarities of ellipticals and associated population deviations (Schweizer et al. 1990; Schweizer \& Seitzer 1992) was revisited. Studying a sample of 117 E classified galaxies we showed that the subsample of 37 with morphological "anomalies", or Pec subsample, had to be sorted out into two families: i) objects in the $Y P$ family, or NGC 2865 type, contain a proportion of stars clearly younger than the old population typical of this Hubble class; ii) objects in the NP family, or NGC 3923 type, do not show evidence of such a young component.

This was indicated by a discussion of the so-called differential colour- or line-indices, i.e. the differences between measured indices and the reference values calculated from the correlations with galaxy mass, as evaluated from the near center velocity dispersion $\log \sigma_{0}$. These correlations have to be traced from the Nop subsample of morphologically "normal" objects, since the inclusion of the Pec subsample introduces an unwanted bias, with the $Y P$ galaxies deviating bluewards (or equivalently for line-indices).
The population indices used in this first paper were the colours $B-V, U-B, B-R, V-I$ and the Lick indices $\mathrm{Mg}_{2}$, $\mathrm{H} \beta,\langle\mathrm{Fe}\rangle, \mathrm{Mg}_{b}$. In Paper II (Michard 2005) the colours $V-J$, $V-K, J-H, J-K$, were added to the data, as derived from the 2MASS images, plus $V$ frames or photometric profiles.

With 12 indices now available for most of the sample, we reconsider the preliminary population models presented in Paper I for two objects only. According to our basic hypothesis, the objects of the NGC 2865 family experienced in a not too distant past a merger plus a starburst. The resulting population can be approximated by the combination of the older one, conveniently described by the $\log \sigma_{0}$ regressions, plus an ad hoc younger SSP (Simple Stellar Population). The SSP is defined by its fractional $V$ luminosity $q_{V}$, age $A$, metallicity $Z$. To derive these parameters from the data the theory of stellar evolution by Bruzual \& Charlot (2003, BC03) is used, with some comparison experiments with Worthey (1994, W94). Such twocomponent models are here derived for 20 objects, mostly classified YP (NGC 2865 family) in Paper I and with sufficient(?) data.

We have also modeled other kinds of ellipticals. The NP galaxies (NGC 3923 family) do not show clear evidence 
of a recent stellar component: they present either the standard E population or a somewhat reddish one, i.e. evidence for higher metallicity. Because of their morphological peculiarities they possibly experienced an episode of star formation (or acquisition), as did $Y P$ objects, but much less recently and with higher $Z$ material available. Attempts were made to describe this postulated event by an SSP but the results are inconclusive.

The Nop subsample is finally considered: the differential indices, i.e. the deviations from the reference regressions, are not exclusively caused by observational errors, but also by variations in the stellar populations leading to correlated changes of the various indices (see Sect. 2.1.1). The sample contains indeed a number of blueish objects, possibly similar to the $Y P$ galaxies of the $P e c$ subsample, and a number of reddisch ones, as found among the $N P$ galaxies. The origin of such fluctuations are considered separately for blueish and reddish objects.

Several workers have estimated the ages of stellar populations in E-type galaxies from the theory of stellar evolution and a small number of selected indices. Bressan et al. (1996) use models with infall (Tantalo et al. 1996), but galaxies are often treated as Simple Stellar Populations (Trager et al. 2000a,b; Kuntschner et al. 2001; Thomas et al. 2002, 2005). The "SSP equivalent age" so derived describes some luminosity weighted mean of the ages of various populations, and accordingly the youngest one is more influential.

Schweizer \& Seitzer (1992) have proposed a "first attempt at dating ancient merger events" from the $U B V$ colours of a sample of E/S0 galaxies. The star formation history of a galaxy formed through a major merger of two spirals ( $\mathrm{Sb}$ or $\mathrm{Sc}$ ) is described by a 4 parameter scheme: one is the unknown age of the event, two of the other are obtained from estimates of the star formation rates before and after a merger, and the last one is varied to provide several possible solutions. The unknown "heuristic merger age" is obtained from the position of the observed object in calculated $U B V$ colour-colour diagrams.

Following Larson \& Tinsley (1978) the ages of the starbursts associated with several merger remnants have been estimated from spectral data (Bernlöhr 1992, 1993a,b; Fritze-v. Alvensleben \& Gerhard 1994; Leonardi \& Rose 1996). These papers deal with rather extreme cases of recent interactions, in contrast to the present work which is concerned with E-type galaxies, so classified in the RC3 and not listed in Arp's Catalogue.

Section 2 summarizes briefly the evidence for population fluctuations among ellipticals, both "peculiar" (Pec subsample in Papers I and II) and "normal" (Nop subsample). It then describes the techniques used to estimate the properties of the SSP "intruders" in the "normal" stellar mix, this being defined by the regressions found in Papers I and II between $\log \sigma_{0}$ and the various indices. The representation by an SSP of the population changes resulting from such a complex event as a merger plus starburst is obviously an over-simplification: the significance of the SSP approximation in the present context is discussed, and the possibility to take into account other parameters, i.e. the presence of dust and the distribution of the new population within the object, is considered. Section 3 gives the results of modeling the objects of the NGC 2865 family with adequate data: for these galaxies, the morphological appearance and the population data suggest our model to be relevant. This may also be true for "normal" blueish objects in the Nop sample. On the other hand, the modeling of reddish objects, both the NP group (NGC 3923 family) and those in the Nop sample, leads to questionable results. In Sect. 4 the significance of our results is discussed in relation to the theories of the build-up of ellipticals through merger events.

Often used notations

- $\sigma_{0}$, near center velocity dispersion;

- $D_{B V}$ : differential colour in $B-V$, i.e. observed colour within the effective radius minus predicted colour from the $\log \sigma_{0}-(B-V)$ relation. Similar notations for other colours and Lick-indices: $D_{U B}, D_{B R}, D_{V I}, D_{V J}, D_{V K}, D_{J K}, D_{\mathrm{Mg} 2}$, $D_{\mathrm{H} \beta}, D_{\langle\mathrm{Fe}\rangle}, D_{\mathrm{Mg} b}$;

- SSP: Simple Stellar Population of unique age and metallicity;

- Pec sample: morphologically peculiar ellipticals; Nop sample: non peculiar objects;

- YP: objects with a detected young population (type NGC 2865); NP objects with no such population (type NGC 3923).

\section{Two-component models of E-type galaxy populations}

\subsection{Principles}

\subsubsection{An ad hoc population index and applications}

To measure coherent population variations in ellipticals, an "Average Differential Index of Population" adip is defined: it is a weighted average of 9 differential colour and line-indices:

$$
\begin{aligned}
\text { adip }=D_{B V}+ & .75 D_{B R}+D_{V I}+.6 D_{V J}+.6 D_{V K} \\
+ & 2 D_{\mathrm{Mg} 2}-0.1 D_{\mathrm{H} \beta}+0.1 D_{\mathrm{Mg} b} .
\end{aligned}
$$

The weights are intended to roughly equalize the influence of the various indices upon the mean. The line indices $D_{\mathrm{H} \beta}$ and $D_{\mathrm{Mg} b}$ are given in $\AA$, hence their small weight. The $D_{\langle\mathrm{Fe}\rangle}$ is not used for reasons to be discussed in Sect. 3. This index has a near-zero value for the Nop sample of 77 objects following the $\log \sigma_{0}$-index relations published in Paper I (Table 6) and Paper II (Table 1), with an average of -0.002 and $\sigma=0.028$. Based on observational errors estimated in Table 1, the standard deviation of individual values of adip may be 0.007 . Figure 1 shows histograms of the adip index separately for the Pec and Nop subsamples. The YP family of blueish objects is clearly apparent in the histogram of "peculiar" galaxies, as well as the several reddish ones in the $N P$ family. The diagram shows a clear minimum near adip $=0$ : it seems that these objects tend to be either too blue or too red.

These two kinds of deviating populations are also present in the histogram of "normal" galaxies, although there are no such extreme cases as NGC 2865 in the blue range of the distribution. The dispersion of the population indices of objects in the Nop sample about the $\log \sigma_{0}$ regressions is far from being due only to errors of measurements: for instance, the standard errors of our $V-K$ data (Paper II) is 0.025 , while the 
Table 1. Estimated mean observational errors $m s e$ for colours and line-indices used in this work. The estimates apply to the sample of circa 100 objects analyzed in Papers I and II and were obtained from multiple observations. Errors for specific objects may be twice the $m s e$ or worse. Errors for $\mathrm{H}_{\beta},\langle\mathrm{Fe}\rangle, \mathrm{Mg}_{b}$ are in $\AA$.

\begin{tabular}{llllll}
\hline \hline Index & $m s e$ & Index & mse & Index & mse \\
\hline$U-B$ & 0.021 & $V-J$ & 0.025 & $\mathrm{Mg}_{2}$ & 0.006 \\
$B-V$ & 0.015 & $V-K$ & 0.025 & $\mathrm{H}_{\beta}$ & 0.12 \\
$B-R$ & 0.020 & $J-H$ & 0.013 & $\langle\mathrm{Fe}\rangle$ & 0.13 \\
$V-I$ & 0.016 & $J-K$ & 0.013 & $\mathrm{Mg}_{b}$ & 0.27 \\
\hline
\end{tabular}
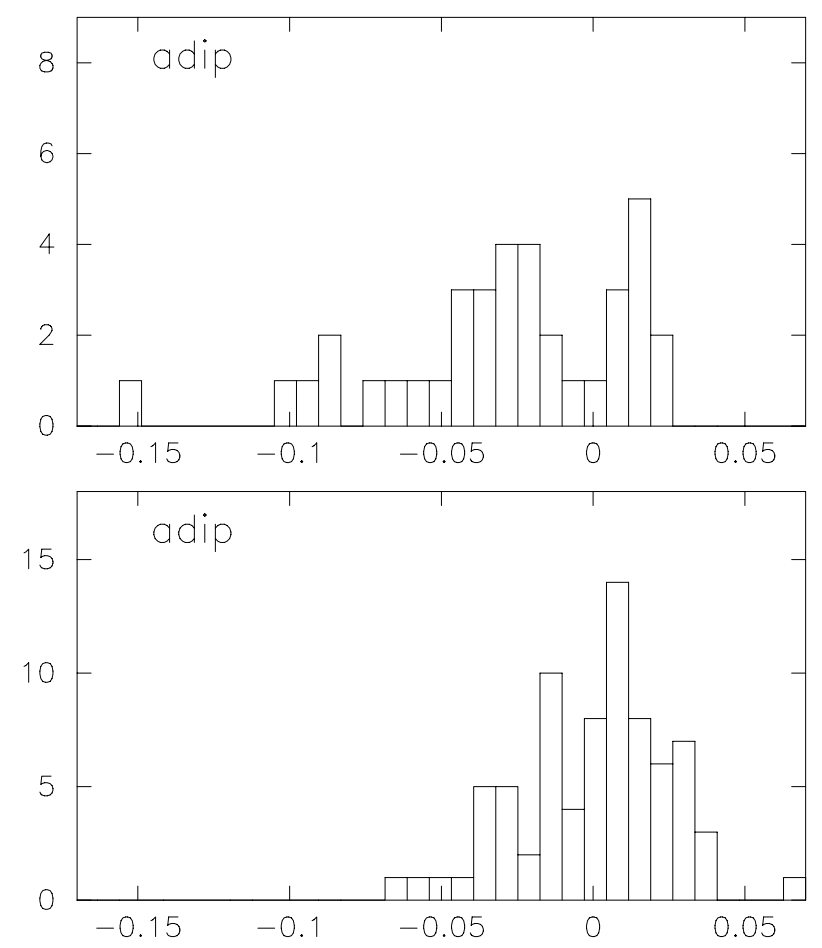

Fig. 1. Histograms for the Pec (upper panel) and Nop (lower panel) subsamples of an ad hoc population index adip incorporating 9 differential colours and line-indices (see definition in text). The near-zero bins contain the objects following the $\log \sigma_{0}$-indices regressions given in Papers I and II.

dispersion about the $\log \sigma_{0}-(V-K)$ regression from the same source reaches 0.071 . Such a "cosmic dispersion" is due to population variations involving coherent changes of various colour and line-indices.

\subsubsection{Principle of two-component models for blueish galaxies}

In the case of galaxies of the NGC 2865 type, we may assume that the morphological peculiarities and the "blueing" of the colours and other population indices, result from the same event. This might be the merger of a pre-existing large earlytype galaxy with a lesser late-type one, resulting in a starburst which will consume most of the ISM of the protagonists. The merger of two spirals of similar masses is not excluded but appears less likely.
Table 2. Estimated errors $\sigma(A)$ in Gyr on ages derived from the $q_{V J K}$ and $q_{\mathrm{Mg} 2}$ ratios. These errors are proportional to $1 / D_{3}$ (see text for definitions), which is here assumed to be -0.1 . (1) $q$ ratio used; (2) age $A$ in Gyr; (3) $\sigma(A)$.

\begin{tabular}{llllll}
\hline \hline Ratio & $A$ & $\sigma(A)$ & Ratio & $A$ & $\sigma(A)$ \\
\hline$q_{V J K}$ & 0.5 & 0.24 & $q_{\mathrm{Mg} 2}$ & 0.5 & 0.18 \\
id & 1.0 & 0.23 & id & 1.0 & 0.23 \\
id & 2.0 & 0.42 & id & 2.0 & 0.25 \\
\hline
\end{tabular}

Both the morphology, described and examplified in Paper I, and the differential indices tabulated in Paper I (Table 7) and Paper II (Table 2) suggest this event to be rather recent as compared to the mean age of the stellar populations in ellipticals, or equivalently of the old population of spirals and S0. The representation of the added stars by an SSP is probably appropriate: the protagonists in the merger contain a mixture of stars of all ages, not likely to deviate much from the assumed primary population, so that the associated starburst is the main source of the juvenile component. These matters will be discussed in more detail below.

In this situation the population of the presently observed galaxy is obtained by summing the old "primary" population with a chosen SSP. The primary has the $\log \sigma_{0}$ of his observed counterpart, that is the mass of the added SSP is deemed negligible. The $V$-luminosity of the primary is unity by definition, and its population indices are obtained from the $\log \sigma_{0}$-index relations previously published in this series. The SSP is defined by its age $A$ and metallicity $Z$ and the corresponding indices taken from BC03 (GALAXEV, version 2003 tables). The versions with the Salpeter IMF and the Padova (1994) stellar tracks were selected. Given the fractional $V$-light luminosity $q_{V}$ of the SSP, the population indices of the mix are readily obtained and also the differential indices to be compared with the observed ones. In the GALAXEV tables, colours and line-indices are given at small steps in age, but at only $6 Z$ values. To model the $Y P$ objects, only 14 age values in the range 5-0.2 Gyr and the 4 metallicities $Z=0.004,0.008,0.02$ and $Z=0.05$ were retained and a model was chosen from the comparison of observations with a grid of $14 \times 4$ predictions.

\subsubsection{The $M / L$ ratio for the "primary" population}

The fractional mass $q_{M}$ of the young component is found from its $q_{V}$ value and the $M / L$ ratios of both components. The $M / L$ of SSP is given in the BC03 tables. To obtain the $M / L$ of the primary (where $M$ is the stellar mass), a number of population mix were considered, each made up of 6 SSP (the 6 available metallicities in $\mathrm{BC} 03$ ) in the range of age 13.5-11 Gyr and with various relative weights: this provided a plausible $(U-V)-M / L$ relation. Through the known $\log \sigma_{0}-(U-V)$ relation (Paper I) the $M / L$ ratios for the "primary" population were derived as $\log M / L=0.227 \log \sigma_{0}+0.268$. 


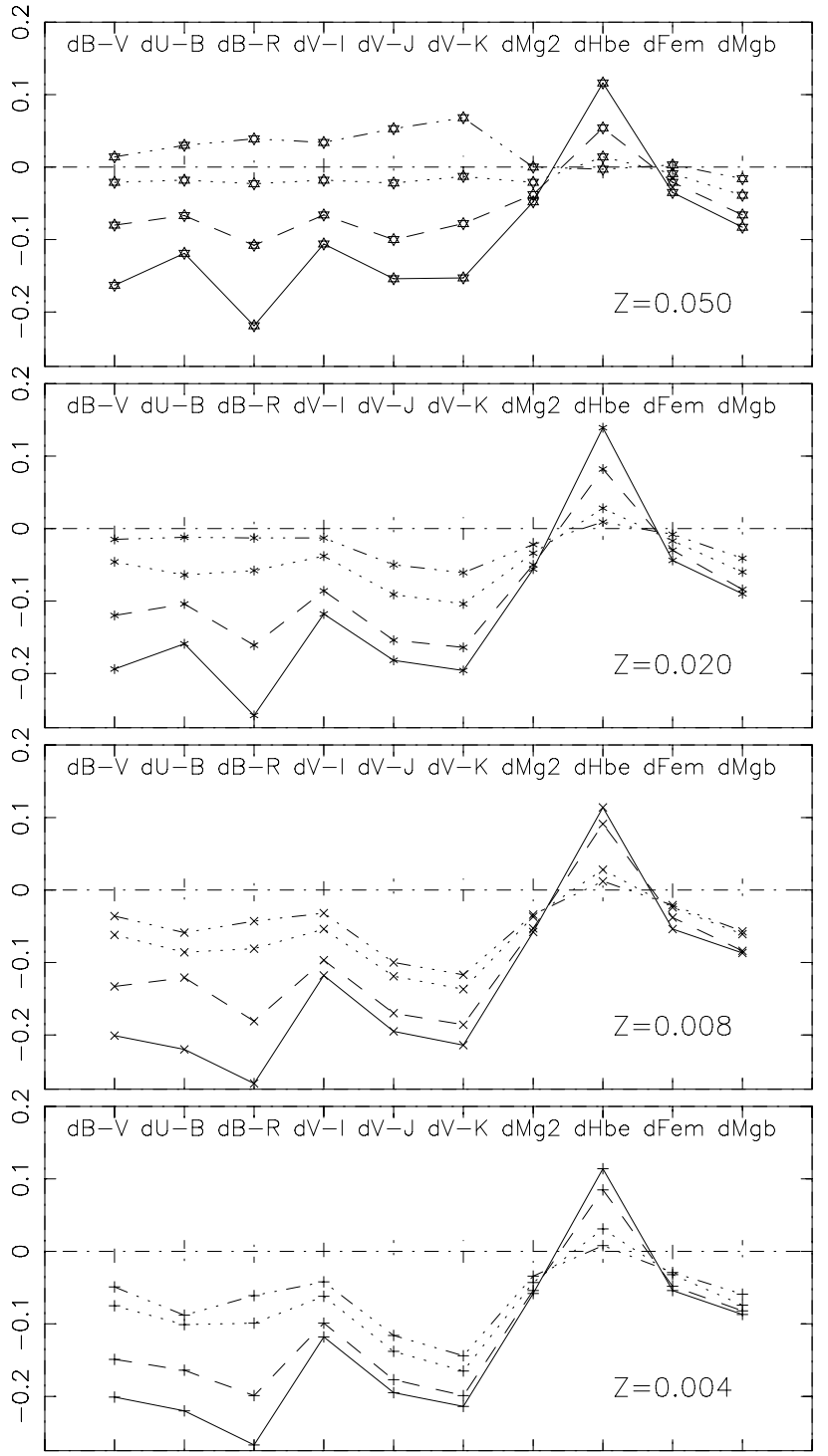

Fig. 2. Calculated differential indices with the BC03 SSP of $V$-luminosity ratio $q_{V}=0.3$ and the primary galaxy with $\log \sigma_{0}=$ 2.35. Abscissae: one step for each index. Ordinates: calculated indices for several ages of the SSP. For clarity, data points for each age $A$ are joined by a line: $0.4 \mathrm{Gyr}$, full line; $0.8 \mathrm{Gyr}$, dashed; $2.0 \mathrm{Gyr}$, dotted; $5.0 \mathrm{Gyr}$, dash-dot-dot-dot. For the Lick indices the unit is $1 / 10 \AA$. At small $A$ the indices are nearly insensitive to $Z$, except its highly supersolar value; this property extends to the full age range for the $\mathrm{H} \beta$ index. Note the variations with age of the $D_{V J}, D_{V K}$ values compared with $D_{B V}, D_{B R}$.

\subsection{Practical aspects}

The $J-H, J-K$ colours are not sensitive enough to the $A$, $Z$ parameters to help in the determination of the model SSP and were discarded. Most of the other differential colours and lineindices help to constrain the parameters $A$ and $Z$ of the juvenile SSP component (and of course $q_{V}$ ). Figure 2 summarizes the variations with $A$ and $Z$ of the differential indices calculated for a primary with $\log \sigma_{0}=2.35$ and the SSP from BC03 with $q_{V}=0.3$. The indices $D_{B R}, D_{V I}$ are nearly proportional to $D_{B V}$ while $D_{V K}$ resembles $D_{V J}$. It is readily apparent that the SSP metallicity has little effect on the differential indices except at large $A$ or for the strongly super-solar $Z=0.05$. Except for this extreme value, $D_{\mathrm{H} \beta}$ is a function of age only.

To find the SSP best matching a modeled galaxy, we first estimate the age from combinations of the observed differential indices empirically found to be age-sensitive. Since all such indices have approximately linear variations with the fractional luminosity $q_{V}$, such combinations must be ratios, the $q_{V}$ parameter being adjusted at a later stage. The chosen ratios may also give an indication of the $Z$ parameter.

The following combinations of differential colours and line-indices have been found useful to locate the "best" SSP in the calculated grid of 56 noted above:

$$
\begin{aligned}
D_{3} & =1 / 3\left(D_{B V}+3 / 4 D_{B R}+D_{V I}\right) \\
q_{V J K} & =1 / 2\left(D_{V J}+D_{V K}\right) / D_{3} \\
q_{\mathrm{Mg} 2} & =D_{\mathrm{Mg} 2} / D_{3} \\
q_{\mathrm{H} \beta} & =0.1 D_{\mathrm{H} \beta} / D_{3}
\end{aligned}
$$

( $D_{\mathrm{H} \beta}$ in $\AA$ ).

$D_{3}$ is an useful substitute for $D_{B V}$ with improved accuracy. Graphs of the useful ratios are collected in Fig. 3. One may guess that $q_{V J K}$ and $q_{\mathrm{Mg} 2}$, especially the first one, are useful indicators of the age of the SSP "intruder". The $Z$ values are less constrained except at relatively old ages, or when the supersolar $Z=0.05$ is indicated by the sign of the differential colours. The solar $Z$ value is adopted if $A<1.5 \mathrm{Gyr}$; at older ages, a perhaps inproved $Z$ is estimated from $q_{\mathrm{Mg} 2}$ and $q_{\mathrm{H} \beta}$. At all stages of the test procedure the luminosity $q_{V}$ is adjusted to the data, in proportion to the adip index. This useful quantity, also used in the histograms of Fig. 1, is defined in Sect. 2.1.1.

As a final approximation, trial and error experiments are performed in a limited range of the parameters in an effort to minimize the least square criterion $\sigma_{\mathrm{O}-\mathrm{C}}$ applied to the O-C residuals of 10 differential indices, all but $D_{J H}$ and $D_{J K}$. The $\mathrm{O}-\mathrm{C}$ (Observed-Calculated) are weighted by the inverse of the observational errors (Table 1 ). In favourable cases we tried to improve the choice of $Z$ by introducing intermediate values in the range $0.02<Z<0.05$ or $0.008<Z<0.02$ : their predicted indices are the averages of those for the adjoining $Z$. They will be noted as $Z=0.032$ for the first range and $Z=0.013$ for the other. Two alternate solutions of similar quality according to the $\sigma_{\mathrm{O}-\mathrm{C}}$ test were sometimes obtained with two different ages from the available grid (assorted with different partial brightnesses): their average $A$ was then selected. A check of the $A-q_{V}$ pair was finally made from the $\mathrm{O}-\mathrm{C}$ of $D_{\mathrm{H} \beta}$.

\subsection{Errors}

\subsubsection{Errors of observational origin}

The observational errors on the colours and line-indices directly apply to the differential indices. The relative errors on these are inversely proportional to their values, that is to the SSP fractional luminosity $q_{V}$ and other SSP parameters. Table 1 gives estimated values of errors on the observed indices, collected from the literature (Poulain \& Nieto 1994; Idiart et al. 2002; Paper II) and from our own discussion of line indices data used in Paper I. We found conflicting evidence as regards 


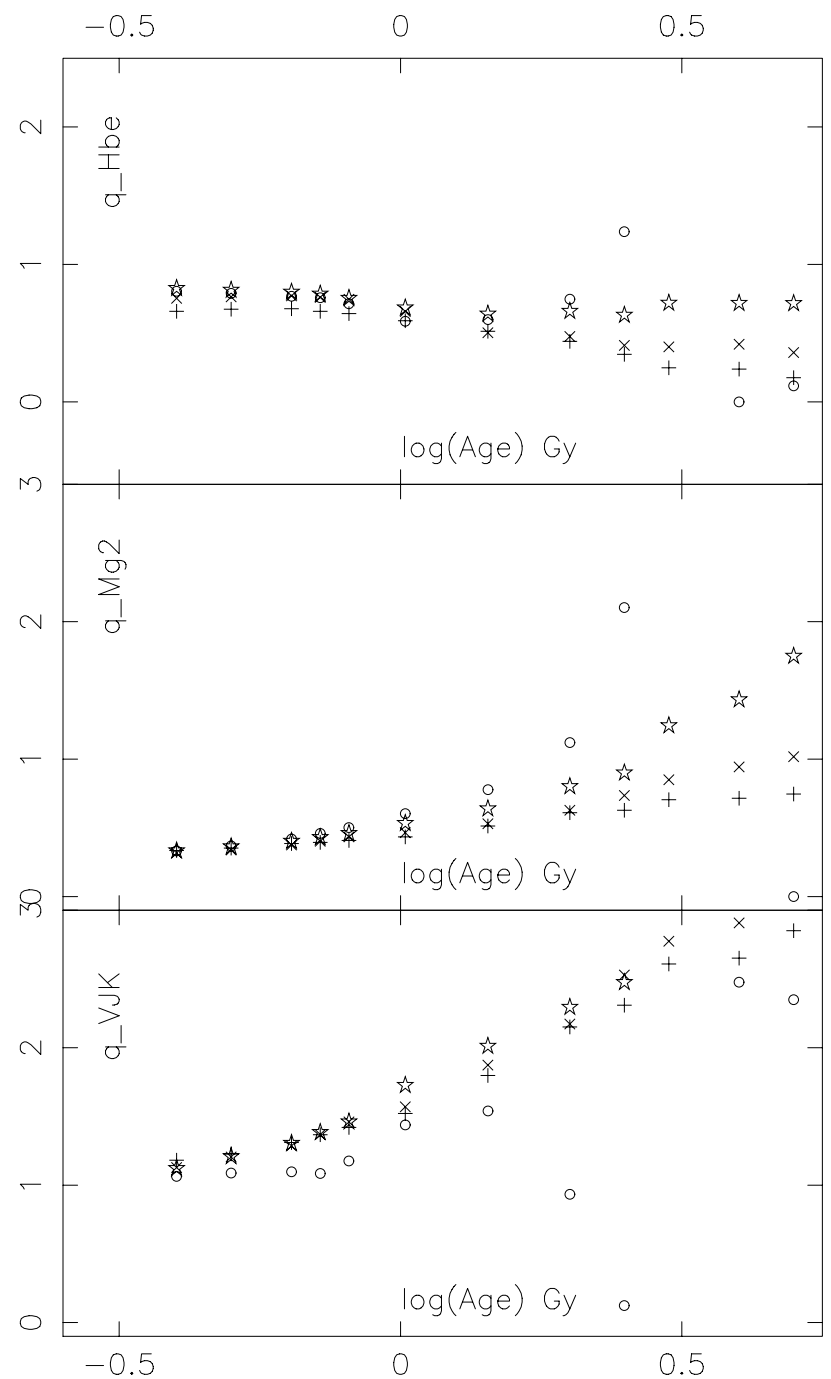

Fig. 3. Three useful ratios in the derivation of two-component models of $Y P$ galaxies with a young population described by a SSP from $\mathrm{BC} 03$. These quantities are defined in the text from the differential indices and are independent of the partial luminosity $q_{V}$. Abscissae: $\log A$ ( $A$ in Gyr). Ordinates: $q$ values with crosses for $Z=0.004, \times$ for $Z=0.008$, stars for $Z=0.02$, circles for $Z=0.05$. The breaks in the curves for $Z=0.05$ are due to the change of sign occuring at large enough $A$ for this metallicity. The ratio $q_{V J K}$ is practically independent of $Z$, hence a good age indicator; the same is true of $q_{\mathrm{Mg} 2}$, sensitive to $Z$ only at $A>2.5 \mathrm{Gyr} ; q_{\mathrm{H} \beta}$ may be used to check $Z$ at $A>2 \mathrm{Gyr}$.

the errors for the $\mathrm{Mg}_{2}$ index. An examination of the data of multiply observed objects in Golev \& Prugniel (1998) would lead to a mean error of 0.005 only. On the other hand we tried to check for the presence of outlyers in the data by considering the correlations between each index and their average adip: two objects with very unlikely values of $D_{\mathrm{Mg} 2}$ were detected (see Table 4). From the errors of various indices, the errors on the ratios $q_{V J H}$ and $q_{\mathrm{Mg} 2}$ are readily evaluated as functions of the "contrast" of the SSP: this is conveniently measured by the $D_{3}$ index which enters in the definition of the two ratios. The errors of age estimates are then found from the $q(A)$ relations, and are summarized in Table 2. It appears that for a relatively high contrast "intruder" with $D_{3}=-0.10$ (near
Table 3. Average O-C of 20 models of $Y P$ galaxies for each of 10 differential population indices: these are near zero, within estimated errors, for most indices, but $D_{U B}$ is systematically "too red" by 0.03 mag and $D_{\mathrm{Mg} b}$ too small by $0.3 \AA$. For $D_{\mathrm{H} \beta}, D_{\langle\mathrm{Fe}\rangle}$ and $D_{\mathrm{Mg} b}$ units are $\AA$.

\begin{tabular}{llcll}
\hline \hline Index & $N$ & O-C & Sigma & Reject \\
\hline$D_{B V}$ & 20 & $0.010 \pm 0.004$ & 0.016 & - \\
$D_{U B}$ & 19 & $0.031 \pm 0.004$ & 0.016 & 3610 \\
$D_{B R}$ & 20 & $-0.008 \pm 0.004$ & 0.020 & - \\
$D_{V J}$ & 20 & $-0.014 \pm 0.005$ & 0.020 & - \\
$D_{V J}$ & 20 & $-0.002 \pm 0.004$ & 0.019 & - \\
$D_{V K}$ & 20 & $0.000 \pm 0.007$ & 0.029 & - \\
$D_{\mathrm{Mg} 2}$ & 20 & $0.000 \pm 0.005$ & 0.021 & - \\
$D_{\mathrm{H} \beta}$ & 15 & $0.03 \pm 0.04$ & 0.18 & - \\
$D_{\langle\mathrm{Fe}\rangle}$ & 16 & $0.11 \pm 0.06$ & 0.25 & - \\
$D_{\mathrm{Mg} b}$ & 16 & $-0.30 \pm 0.10$ & 0.38 & - \\
\hline
\end{tabular}

the observed $D_{3}$ for NGC 5018, 5061 for instance) the errors of ages derived from either one of the two ratios are about $0.2-0.25 \mathrm{Gyr}$ for $A=1 \mathrm{Gyr}$. The situation is less satisfactory for objects with a juvenile population of a much lesser contrast, such as NGC 1549 with $D_{3}=0.015$ only. Then the existence of more than one age indicators is needed to reach a significant result.

\subsubsection{Errors from theoretical inadequacies}

If a set of differential indices is calculated for the injection of an SSP from the $\mathrm{BC} 03$ tables, their relative values are fixed, being a property of the stellar evolution theory used. These ratios are slightly dependent on the $A$ and $Z$ SSP parameters. In the fitting of models we try to minimize a function of 10 differential indices, i.e. $\sigma_{\mathrm{O}-\mathrm{C}}$. Inadequacies of the SSP models may lead to systematic non-zero values for one or the other of each of the $\mathrm{O}-\mathrm{C}$ residuals, even if the $\sigma_{\mathrm{O}-\mathrm{C}}$ is minimal.

A test uses the models obtained for the 20 objects of the NGC 2865 family (Table 4). In Table 3 are presented the average $\mathrm{O}-\mathrm{C}$ for each of the 10 population indices influential in the modeling. Near zero values are obtained for most indices (as hoped). The average $\mathrm{O}-\mathrm{C}$ is generally less or much less than the associated $\sigma$ except for $D_{U B}$ and $D_{\mathrm{Mg} b}$. The $U-B$ colour is 0.033 mag redder on average than expected from the other indices in the same object; $D_{B V}$ is also slightly too red while $D_{B R}$ and $D_{V I}$ are slightly too blue. Finally, $D_{\mathrm{Mg} b}$ is too small by $0.3 \AA$ while $D_{\langle\mathrm{Fe}\rangle}$ is too large by $0.1 \AA$.

This difficulty can also be studied by using SSP from another source, preferably based on different foundations from BC03. We have chosen the SSP of W94 because they entirely fulfill this condition, both as regards the evolutionary tracks and the spectral library. Unfortunately their lower threshold in age is $1.5 \mathrm{Gyr}$, limiting the possibilities for comparison. Figure 4 compares models of two ages for the object with $\log \sigma_{0}=2.35$ polluted by an SSP of solar luminosity, i.e. $Z=0.02$ for $\mathrm{BC} 03$ and $[\mathrm{Fe} / \mathrm{H}]=0$ for W94. There are important divergences between the predicted differential indices 
Table 4. SSP models of population anomalies: the $Y P$ (or NGC 2865) family in the Pec sample, including 2 objects classified as uncertain in Paper I. Two models, one without dust the other with some dust, are given for NGC 1700, 3610 and 5018, while only a dusty model is feasible for IC 3370. (1) Name; (2) $\log \sigma_{0}$ velocity dispersion; (3) $\Sigma_{2}$ peculiarity index (Paper I); (4) adip observed average differential index; (5) $f I R$ far IR index, i.e. $100 \mu$ flux in Knapp et al. (1989) corrected to the distance of the Virgo Cl. (6) $\tau_{V}$ optical depth of dust (Tau(V) in Wal92 notation); (7) $A$ age of model SSP in Gyr; (8) $Z$ metallicity; (9) $q_{V}$ fractional $V$ luminosity; (10) $q_{M}$ fractional mass in $\%$; (11) data: nLi missing Lick indices, ?? see notes below; (12) aeq age estimate quality, i.e. number of criteria giving an $\mathrm{O}-\mathrm{C}$ within obervational errors. These may be $q_{V J K}, q_{\mathrm{Mg} 2}, D_{\mathrm{H} \beta}$; (13) $\sigma_{\mathrm{O}-\mathrm{C}}$ quadratic mean of the $\mathrm{O}-\mathrm{C}$ of 10 differential population indices, all in mag and weighted by the inverse of average observational errors. Miscellaneous notes: NGC 1653 no $\mathrm{H} \beta$. NGC 4374, $4406 \mathrm{H} \beta$ rejected. NGC 3610: $U-B$ rejected. NGC 3557 , 5557 anomalous $\mathrm{Mg}_{2}$. NGC 5322 anomalous $V-I$.

\begin{tabular}{|c|c|c|c|c|c|c|c|c|c|c|c|c|}
\hline (1) & (2) & (3) & (4) & $(5)$ & (6) & (7) & (8) & (9) & (10) & (11) & (12) & (13) \\
\hline Name & $\log \sigma_{0}$ & $\Sigma_{2}$ & adip & $f I R$ & $\tau_{V}$ & $A$ & $Z$ & $q_{V}$ & $q_{M}$ & Data & aeq & $\sigma_{\mathrm{O}-\mathrm{C}}$ \\
\hline NGC 0596 & 2.21 & 2.1 & -0.030 & 0 & 0.0 & 1.02 & 0.020 & 0.09 & 1.2 & - & 2 & 1.28 \\
\hline NGC 1549 & 2.33 & 7.0 & -0.016 & 130 & 0.0 & 2.00 & 0.013 & 0.09 & 2.0 & - & 2 & 0.89 \\
\hline NGC 1653 & 2.40 & 3.5 & -0.046 & 4100 & 0.0 & 2.50 & 0.013 & 0.23 & 5.9 & $? ?$ & 2 & 1.16 \\
\hline NGC 1700 & 2.39 & 3.8 & -0.057 & 0 & 0.0 & 0.40 & 0.020 & 0.08 & 0.5 & - & 2 & 1.46 \\
\hline id & id & id & id & id & 0.25 & 0.64 & 0.020 & 0.12 & 1.0 & - & 3 & 1.47 \\
\hline NGC 2865 & 2.25 & 10.6 & -0.156 & 1920 & 0.0 & 0.81 & 0.020 & 0.60 & 6.7 & - & 3 & 1.27 \\
\hline NGC 3557 & 2.42 & 4.3 & -0.089 & 2720 & 0.0 & 1.02 & 0.013 & 0.25 & 2.7 & nLi ?? & 1 & 1.97 \\
\hline NGC 3585 & 2.35 & 2.8 & -0.029 & 0 & 0.0 & 1.72 & 0.013 & 0.13 & 2.4 & - & 2 & 1.03 \\
\hline NGC 3610 & 2.21 & 6.8 & -0.049 & 730 & 0.0 & 0.29 & 0.032 & 0.09 & 0.5 & $? ?$ & 2 & 1.35 \\
\hline id & id & id & id & id & 0.5 & 0.50 & 0.032 & 0.16 & 1.4 & id & 2 & 1.35 \\
\hline NGC 3613 & 2.33 & 1.7 & -0.031 & & 0.0 & 3.00 & 0.013 & 0.24 & 7.3 & - & 3 & 1.13 \\
\hline NGC 4125 & 2.38 & 2.5 & -0.033 & 3890 & 0.0 & 2.50 & 0.008 & 0.18 & 4.1 & - & 3 & 1.22 \\
\hline NGC 4374 & 2.47 & 2.1 & -0.033 & 1020 & 0.0 & 3.00 & 0.013 & 0.26 & 7.5 & $? ?$ & 2 & 1.51 \\
\hline NGC 4406 & 2.40 & 3.1 & -0.033 & & 0.0 & 5.00 & 0.013 & 0.14 & 6.6 & $? ?$ & 2 & 1.08 \\
\hline NGC 4976 & 2.20 & 5.2 & -0.104 & 0 & 0.0 & 0.81 & 0.020 & 0.36 & 4.1 & $\mathrm{nLi}$ & 1 & 2.09 \\
\hline NGC 5018 & 2.33 & 8.0 & -0.081 & 5880 & 0.0 & 0.50 & 0.020 & 0.15 & 1.1 & - & 1 & 2.28 \\
\hline id & id & $\mathrm{id}$ & id & id & 0.5 & 0.81 & 0.032 & 0.32 & 3.8 & - & 3 & 1.69 \\
\hline NGC 5061 & 2.29 & 3.2 & -0.088 & 0 & 0.0 & 0.72 & 0.020 & 0.25 & 2.5 & - & 2 & 1.41 \\
\hline NGC 5322 & 2.36 & 2.6 & -0.040 & 2690 & 0.0 & 0.68 & 0.020 & 0.08 & 0.7 & $? ?$ & 2 & 1.51 \\
\hline id & id & id & & id & 0.25 & 1.02 & 0.020 & 0.12 & 1.5 & id & 3 & 1.12 \\
\hline NGC 5557 & 2.41 & 6.7 & -0.045 & 0 & 0.0 & 0.72 & 0.020 & 0.10 & 0.9 & $? ?$ & 2 & 1.50 \\
\hline NGC 5576 & 2.27 & 8.0 & -0.065 & 350 & 0.0 & 2.00 & 0.013 & 0.35 & 7.9 & - & 2 & 1.38 \\
\hline NGC 5982 & 2.40 & 6.8 & -0.050 & 1810 & 0.0 & 1.23 & 0.013 & 0.16 & 2.1 & - & 3 & 0.85 \\
\hline IC 3370 & 2.39 & 7.7 & -0.014 & 8460 & 1.0 & 0.72 & 0.020 & 0.12 & 1.2 & $\mathrm{nLi}$ & 1 & 0.60 \\
\hline
\end{tabular}

from the two SSP sources: the $D_{U B}$ is bluer with W94, the other colours redder; the line indices are in good agreement. Two-component models have been calculated for 10 galaxies, using both the W94 and BC03 SSP tables. The results are in rather good agreement: the rms of the differences in estimated ages is 0.35 Gyr and 0.005 for the estimated $Z$. As regards the systematic trends in average $\mathrm{O}-\mathrm{C}$ values for each index found above with BC03 for $D_{U B}$ and $D_{\mathrm{Mg} b}$ (Table 3), they also occur with the W94 SSP tables and are the same within uncertainties; the small mean residual found for $D_{\langle\mathrm{Fe}\rangle}$ nearly reduces to zero.

The systematic divergences from zero of the $\mathrm{O}-\mathrm{C}$ values for some differential indices might also result from errors in the assumed indices of the primary, i.e. in the assumed regressions of these against $\log \sigma_{0}$. Looking at the corresponding correlation diagrams of Papers I and II, the assumption of a linear relation may be sometimes uncertain: for instance one would be tempted to introduce a curvature in the relation between $U-B$ and $\log \sigma_{0}$.

\subsubsection{Location of star sampling in observations}

The differential colour indices are sampled in a cylindrical volume of the galaxy under study limited by its de Vaucouleurs radius $r_{\mathrm{e}}$, while the Lick indices are sampled through a central small aperture, expressed in arcsec, and not the same in the various data sources used in our compilation: for one of these only, the Lick indices are corrected to an $r_{\mathrm{e}} / 8$ aperture (Trager et al. 2000a). The indices are used concurrently in the derivation of the 3 parameters defining the intruding SSP, leading to possible uncertainties. The $A, Z$ parameters of the added population are possibly nearly the same close to the center and within $r_{\mathrm{e}}$, but its fractional mass and luminosity are eventually 


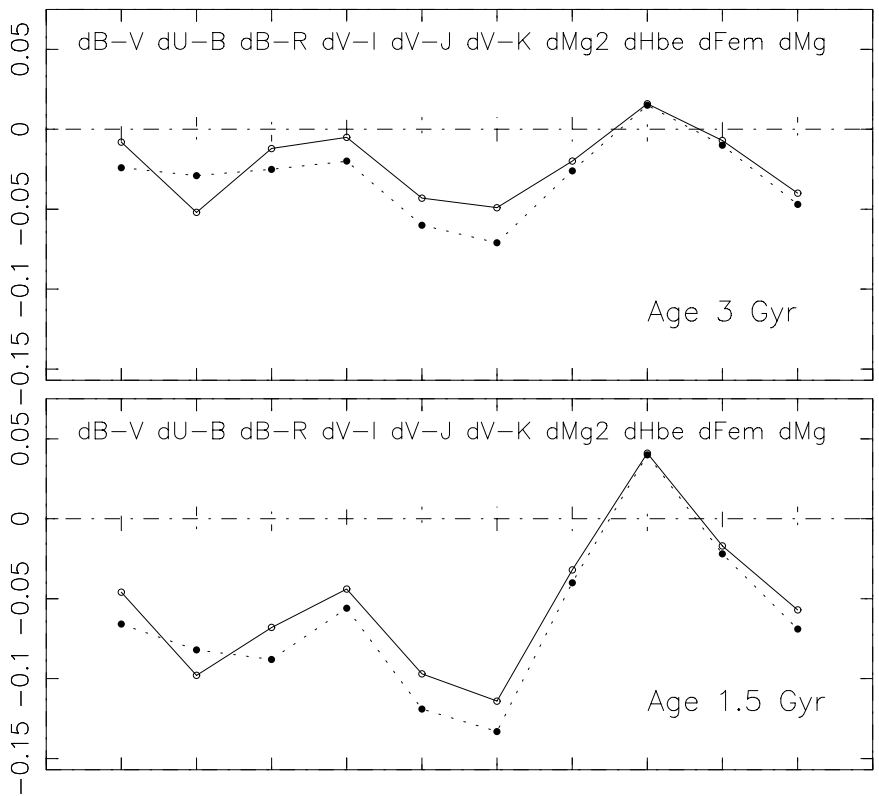

Fig. 4. Comparisons of two-component models involving a standard primary with $\log \sigma_{0}=2.35$ and SSP built from BC03 and W94. Abscissae: one step for each used differential index. Ordinates: differential indices in mag, or for Lick indices in tenths of $\AA$. For clarity, data points for each SSP source are joined by a line: W94, full line joining open circles. BC03, dotted line joining keavy dots. Lower panel: age $1.5 \mathrm{Gyr}$; Upper panel: age $3 \mathrm{Gyr}$.

different. Then an optimal model would involve different $q_{V}$ parameters for the colour- and line- indices respectively (and consequently $q_{M}$ ). It is likely that the "new" stars from the starburst are concentrated towards the center of the object. Larger $q_{V}$ parameters for line-indices than for colours are then needed to improve the model as will be verified below.

\subsubsection{The $[\alpha / \mathrm{Fe}]$ problem}

It was shown long ago (Worthey et al. 1992) that the $\langle\mathrm{Fe}\rangle$ Lick indices are very poorly correlated with $\mathrm{Mg}_{b}$, and with a slope much smaller than expected from the W94 calculations for SSP of solar chemistry. It has been assumed that a third parameter is needed, besides $A$ and $Z$, to describe the populations of ellipticals. This may be $[\mathrm{Mg} / \mathrm{Fe}]$ or $[\alpha / \mathrm{Fe}]$ (Tantalo \& Chiosi 2002; Thomas et al. 2005) or [E/Fe] (Trager et al. 2000b). In our compiled data the relation between $\langle\mathrm{Fe}\rangle$ and $\mathrm{Mg}_{b}$ is similar to graphs published by Worthey et al. (1992) or Kuntschner et al. (2001), and the differential indices $D_{\langle\mathrm{Fe}\rangle}$ and $D_{\mathrm{Mg} b}$ are almost uncorrelated. The values of $D_{\langle\mathrm{Fe}\rangle}$ does not contribute significantly to the derivation of our models, because they are small compared to errors. On the other hand it appears from Table 3 above that the measured $D_{\langle\mathrm{Fe}\rangle}$ are larger than modeled by $0.1 \AA$ on average, while the $D_{\mathrm{Mg} b}$ are too small by $0.3 \AA$. This might suggest that the younger stars added to old Es in recent interactions have more $\mathrm{Fe}$ and less $\mathrm{Mg}$ than in the primary population. Such systematic changes in $[\alpha / \mathrm{Fe}]$ might perhaps play some role in the trends of other $\mathrm{O}-\mathrm{C}$ residuals (see Sect. 2.3.2), notably $D_{U-B}$.

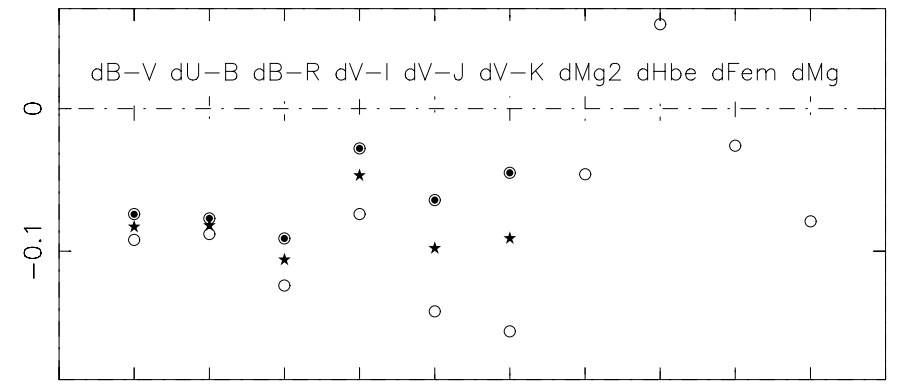

Fig. 5. Influence of diffuse dust on a two-component model involving a standard primary with $\log \sigma_{0}=2.35$ and an SSP with $A=1 \mathrm{Gyr}, Z=$ 0.02 and $q_{V}=0.3$. The dust reddening is evaluated from Witt et al. (1992, Wa192), Table 3E. Abscissae: one step for each used differential index. Ordinates: no dust (open circles); $\mathrm{Tau}(V)=0.5$ in Wal92 (black stars); $\operatorname{Tau}(V)=1$. (big filled circles). For Lick indices, ordinates are expressed in tenths of $\AA$.

\subsubsection{The problem of dust}

The presence of some dust in the studied galaxies will change the differential colour indices, very little for $D_{U B}$, very much for $D_{V K}$. Dust effects are illustrated in Fig. 5 for a calculated model with a primary object of $\log \sigma_{0}=2.35$ and an SSP with $A=1 \mathrm{Gyr}, Z=0.02$ and $q_{V}=0.3$. The relative colour excesses are obtained from Witt et al. (1992), or Wal92: they give the emergent light in various pass-bands at the center of a spherical galaxy following approximately the $r^{1 / 4}$ law, with various amounts of dust measured by the central $V$ optical depth $\operatorname{Tau}(V)$. In this discussion we adopt as reddening of the observed colours within $r_{\mathrm{e}}$ the central colours found from Wal92.

As seen from Fig. 5 an average dust amount $(\operatorname{Tau}(V)=1$ in the Wal92 scale) changes $U-B$ by 0.011 and $B-V$ by 0.018 but the reddening reaches 0.12 in $V-K$. The ratios $q_{V J K}$ and $q_{\mathrm{Mg} 2}$ used as age indicators will be modified, from 1.73 to 0.88 and 0.82 to 0.60 respectively. The previous agreement of the corresponding ages is destroyed. The disagreement between colours and Lick indices population indicators allows the detection of dust in observed galaxies as seen below.

\section{Results of modeling}

\subsection{Results for the YP family (NGC 2865)}

Models have been obtained for 20 blueish objects (out of 26) classified in Paper I as YP (NGC 2865 family), or $Y P$ : or NP: i.e. uncertain. NGC 1344 and NGC 3640 could not be treated for lack of the $D_{V J}, D_{V K}$ indices, crucial for age estimates. Others had too incomplete or inconsistent data.

Some essential parameters of the observed object and the 3 parameters defining the SSP "intruder" are given in Table 4. The following remarks may apply:

1. Figure 6 shows the 10 useful differential indices for the prototypical object NGC 2865, its adopted model and the corresponding $\mathrm{O}-\mathrm{C}$ residuals. These are reasonably small, and close to the average residuals of Table 3: some of these are not zero, possibly as a property of the BC03 models 


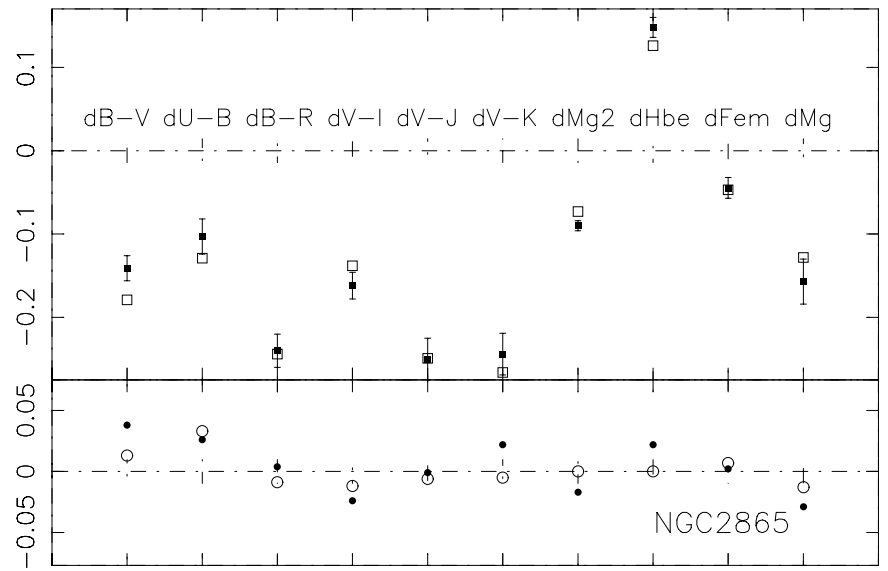

Fig. 6. Two component model for NGC 2865. Abscissae: one step for each used differential index. Ordinates in upper panel: observed differential indices (filled squares) and calculated values from model in Table 4 (open squares). Lower panel: $\mathrm{O}-\mathrm{C}$ of differential indices (full dots) compared to the average of $\mathrm{O}-\mathrm{C}$ for 20 models of $Y P$ galaxies as given in Table 3 (open circles). For Lick indices, ordinates are expressed in tenths of $\AA$. The model is successful for all population indices.

(see Sect. 2.3.2). We suggest that our model represents correctly the mean population of NGC 2865 . Graphical representations of models for other objects of the same family are shown in Figs. 7 and 8.

2. The distribution of ages in the accepted interval $(0.2<$ $A<5$ Gyr) is not uniform. There are 12 recent "intruders" $(A \leq 1$. Gyr) and 6 old one $(A \geq 2$. Gyr) with only two in between. Objects with young SSP components show morphological evidence of recent mergers, with two exceptions, NGC 5322 and NGC 1700. Objects with older SSP are affected by asymmetries, without spectacular morphological peculiarities: an exception is NGC 5576 (see the displays in Paper I, electronic edition, for this and other galaxies).

3. For reasons given above, the $Z$ parameter generally has the solar value for recent "intruders". An interesting exception is NGC 3610, with the youngest population of the 20 models $(A=0.29 \mathrm{Gyr})$, where a super-solar value is indicated. On the other hand older SSP "intruders" may have subsolar $Z$ values. Note that the choice of a non-solar SSP relies on minute variations of the $\sigma_{\mathrm{O}-\mathrm{C}}$ test.

4. The relative masses of SSP "intruders", in terms of the mass of the "primary", varies from $0.5 \%$ (NGC 1700) to $7.9 \%$ (NGC 5576). The average mass for the 11 younger events $(A \leq 1.0 \mathrm{Gyr})$ is $2.2 \%$, while it reaches $5.1 \%$ for the 9 older $(A \geq 1.0 \mathrm{Gyr})$. Obviously, the old events should have been more important to produce population changes large enough to remain in the range of possible detection.

5. The colours of a few objects are possibly influenced by the presence of dust, and the corresponding dusty models were derived: these were selected from the inspection of the O-C of differential indices and from the values of the IRAS $100 \mu$ flux, larger than 2000 in the Knapp et al. (1989) catalogue, after correction to the standard distance of the Virgo cluster. From the 8 derived dusty models, those

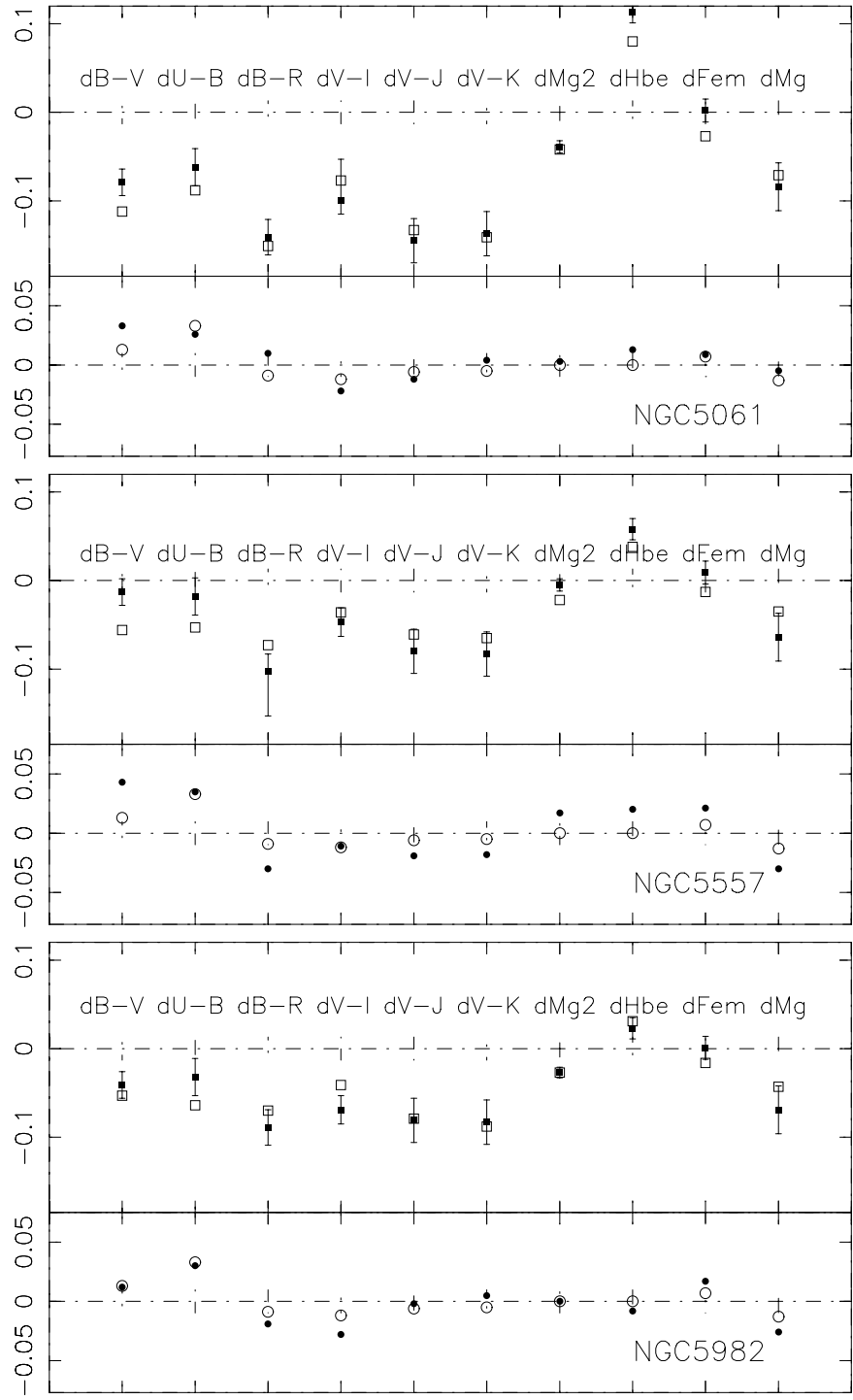

Fig. 7. Two component models for several objects of the NGC 2865 family and an added population younger than $1 \mathrm{Gyr}$. Conventions are the same as in Fig. 6. The model parameters are given in Table 4.

for NGC 1653, 3557, 4125 were less good than the dustless models and are not published. Models for NGC 1700 and 3610, two objects with modest far-IR fluxes, are possible alternatives to the dustless models and are entered in Table 4. The same is done for the dusty models of NGC 5018 and 5322, distinctly better than the dustless ones, as judged from the $\sigma_{\mathrm{O}-\mathrm{C}}$ values: these objects are rather bright at $100 \mu$. An interesting case is IC 3370, a bright object in the far-IR, with no possible solution without dust, because the $U B V R I$ differential indices and $D_{\mathrm{Mg} 2}$ indicate the presence of a young population while the $D_{V J}$ and $D_{V K}$ do not: a good solution has been obtained with a rather large amount of dust. Lick indices are unfortunately missing. Graphical representations are given in Fig. 9 for 3 of the dusty models. We did not devote much effort to ascertain the optimal amount of dust in these models, and no interpolation between the $\operatorname{Tau}(V)$ values in Wal92 was attempted. The colour excesses of Wal92 for their model 


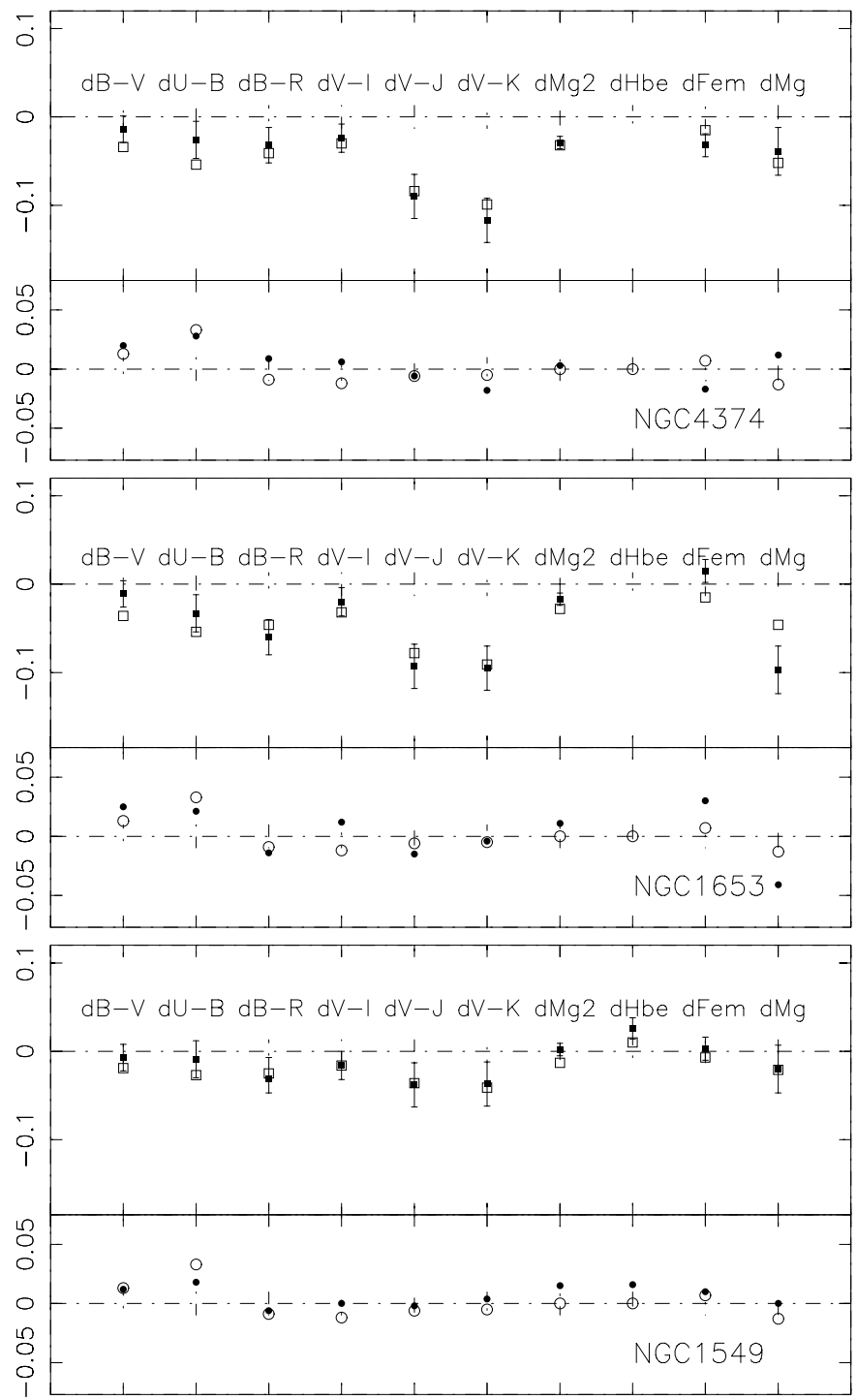

Fig. 8. Two component models for objects with an added population older than 2 Gyr. Conventions are the same as in Fig. 6. The model parameters are given in Table 4.

with a $V$ central dust optical depth $\operatorname{Tau}(V)$ between 0.25 and 1.0 were retained. Since dust mitigates the "blueing" of the young population, the dusty models involve a redder, i.e. older SSP population than the "clean" one of the same galaxy.

6. As indicated above, the concentration of the younger stars may be higher towards center than in the $r_{\mathrm{e}}$ domain so that different $q_{V}$ (and $q_{M}$ ) parameters may be needed for lineand colours differential indices, here noted respectively $q 1_{V}$ and $q 2_{V}$. This probably occurs in a few of the studied objects, such as NGC 2865 and 5018 (see Figs. 6 and 9): our solutions lead to observed $D_{B V}$ and other colours slightly too red, while $D_{\mathrm{Mg} 2}, D_{\mathrm{Mg} b}, D_{\mathrm{H} \beta}$ are slightly too blue as compared to the model. The previously calculated models have been modified with the introduction of two $q_{V}$ values, leading to the following results: i) for NGC 2865, with $q 1_{V}=0.80$ and $q 2_{V}=0.55$ the $A, Z$ model of Table 4 is improved to a $\sigma_{\mathrm{O}-\mathrm{C}}=0.96$ instead of 1.27. The mass

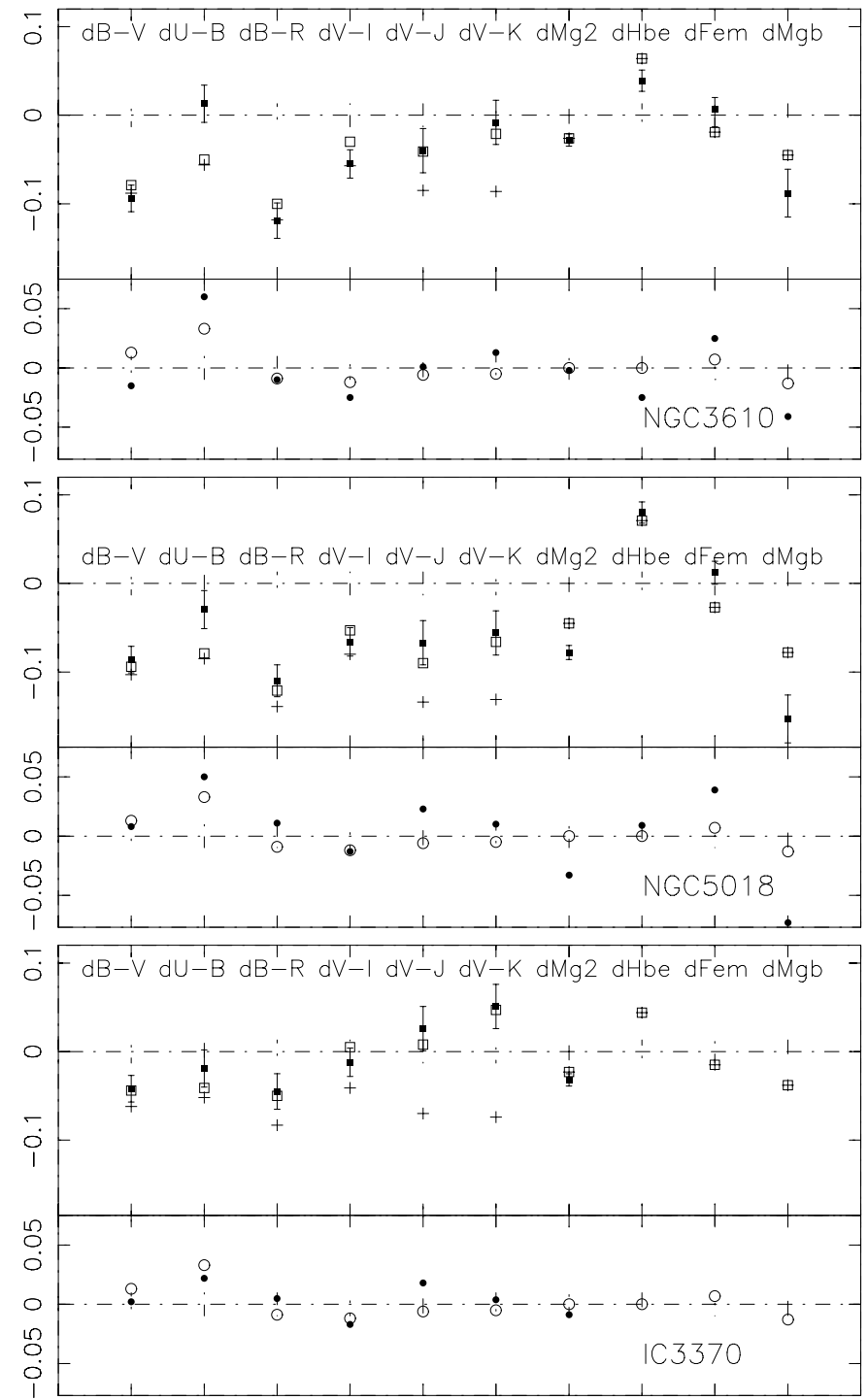

Fig. 9. Two component models for objects with a juvenile population and a notable amount of dust, from far IR fluxes and population indices. Ordinates in upper panel: observed differential indices (filled squares); colours corrected for dust (open crosses); calculated values from model in Table 4 (open squares). Lower panel: $\mathrm{O}-\mathrm{C}$ of differential indices (full dots) compared to the average of $\mathrm{O}-\mathrm{C}$ for 20 models of $Y P$ galaxies as given in Table 4.

concentration of the young SSP component is $9 \%$ in the near center region instead of $6.2 \%$ in the average within $r_{\mathrm{e}}$; ii) for NGC 5018, the dusty model of Table 4 and Fig. 9 is improved by using $q 1_{V}=0.47$ and $q 2_{V}=0.28$. In terms of the $\sigma_{\mathrm{O}-\mathrm{C}}$ test the improvement from 1.69 to 1.55 is modest, due to the inconsistency of the various line-indices: the observed $D_{\mathrm{Mg} 2}, D_{\mathrm{Mg} b}$ are smaller than expected and the $D_{\langle\mathrm{Fe}\rangle}$ larger, i.e. the young added stars are Fe-rich in this object. The mass concentration of the young SSP component is $5.5 \%$ in the near center region against $3.2 \%$ in the full $r_{\mathrm{e}}$ domain. 
Table 5. SSP models of population anomalies: blueish objects in the Nop sample. Same disposition as Table 4.

\begin{tabular}{llllllllllllll}
\hline \hline$(1)$ & $(2)$ & $(3)$ & $(4)$ & $(5)$ & $(6)$ & $(7)$ & $(8)$ & $(9)$ & $(10)$ & $(11)$ & $(12)$ & $(13)$ \\
Name & $\log \sigma_{0}$ & $\Sigma_{2}$ & adip & $f I R$ & $\tau_{V}$ & $A$ & $Z$ & $q_{V}$ & $q_{M}$ & Data & aeq & $\sigma_{\mathrm{O}-\mathrm{C}}$ \\
\hline NGC 0584 & 2.35 & 0.0 & -0.039 & 1000 & 0.0 & 2.00 & 0.013 & 0.19 & 4.1 & - & 3 & 1.24 \\
NGC 1600 & 2.54 & 0.0 & -0.015 & 2390 & 0.0 & 3.00 & 0.013 & 0.09 & 2.5 & - & 2 & 1.03 \\
NGC 2434 & 2.32 & 0.0 & -0.049 & 0 & 0.0 & 1.43 & 0.020 & 0.18 & 3.2 & - & 3 & 1.05 \\
NGC 3087 & 2.35 & 0.0 & -0.065 & 0 & 0.0 & 1.02 & 0.013 & 0.19 & 2.2 & - & 2 & 1.82 \\
NGC 3250 & 2.42 & 0.0 & -0.047 & 0 & 0.0 & 1.02 & 0.020 & 0.20 & 2.4 & nLi & 1 & 1.97 \\
NGC 3377 & 2.16 & 0.0 & -0.039 & 120 & 0.0 & 0.81 & 0.020 & 0.11 & 1.3 & - & 3 & 1.10 \\
NGC 4168 & 2.28 & 0.0 & -0.029 & 2620 & 0.0 & 3.00 & 0.013 & 0.24 & 7.6 & - & 2 & 1.92 \\
NGC 4278 & 2.39 & 0.0 & -0.032 & 1700 & 0.0 & 2.50 & 0.013 & 0.21 & 5.4 & $\mathrm{nLi}$ & 1 & 1.17 \\
NGC 4478 & 2.16 & 0.0 & -0.038 & 0 & 0.0 & 2.50 & 0.013 & 0.30 & 8.8 & - & 3 & 1.25 \\
\hline
\end{tabular}

\subsection{Results for other objects}

\subsubsection{The blueish objects in the Nop subsample}

As apparent from Fig. 1, there are among mophologically normal ellipticals, "blueish" objects and "reddish" objects similar to the $Y P$ and NP families of the Pec sample. The blueish objects may be described by two-component models as proposed for peculiar objects of the $Y P$ family or similar. The solutions for 9 blueish objects are given in Table 5. These have been primarily selected from the index adip $<-0.03$ ( 7 cases), with 2 limiting cases added. The ages and other parameters of the SSP "intruder" fall in the same intervals as found above for the YP galaxies of Pec sample. However the proportion of "old" ( $A \geq 2$. Gyr) and "recent" $(A \leq 1$. Gyr) intruders are reversed. Only one of the postulated events is younger than $1 \mathrm{Gyr}$. This seems to have occured in the well-known disky galaxy NGC 3377, but did not leave morphological peculiarities (see Paper I). We have again tried to improve the models by "injecting" some dust in two objects with $100 \mu$ flux larger than 2000, i.e., NGC 1600 and 4168, but these models are not better than the dustless ones and are not published. The present interpretation of blueish galaxies in the Nop sample is uncertain, since the morphological evidence for an interaction event is lacking.

\subsubsection{The reddish galaxies in the Pec and Nop samples}

As seen from Fig. 1 the $P e c$ sample contains several reddish galaxies members of the $N P$ family. Among these we note NGC 3923, a famous shell galaxy, 5846 with a remarkable asymmetry (see Paper I) and 2974, rather dusty from IRAS data. Similarly, there are in the Nop sample a number of galaxies redder than expected from errors in population indices; 5 objects with adip $>0.02$ were selected for model testing.

For these objects the reddening requires the acquisition of strongly super-solar stars $(Z=0.050)$ in the distant past, $A=4$ to $10 \mathrm{Gyr}$, and involving partial masses of the order of $10 \%$ or more, i.e. an important event. The $q_{M}$ parameter here may be too large to be considered a small fraction of the primary object as assumed in our working hypothesis (see Sect. 2.1). On the other hand, the age of the super-solar SSP needed to represent the extra-population of these reddish objects cannot be fixed in the range 4-10 Gyr: any age can match the data with a proper choice of the partial luminosity $q_{V}$. Tentative models of reddish objects also have been obtained with the SSP from W94. Since these tables extend to a higher super-solar metallicity than $\mathrm{BC} 03$, up to $[\mathrm{Fe} / \mathrm{H}]=0.50$, higher reddenings are obtained at smaller $q_{M}$ values. Degeneracy in the parameters $A$ (in the range 3-10 Gyr) and $q_{V}$ (or $q_{M}$ ) also occurs and precludes an actual datation of "events" perhaps associated with the morphological peculiarities of red objects in the NGC 3923 family.

The presence of diffuse dust is obviously a likely explanation for E-galaxies redder than average. As above (see Sect. 3.1 and Fig. 9) IRAS data were used to find possible dusty objects. Line-indices are unchanged by the presence of dust while the colour excess should be larger and larger going from $D_{U B}$ to $D_{V K}$ : we have tried to use this property to locate dusty Es. Taking into account the limitations of both data, these two approaches lead to convergent results, although not to a good agreement. For 19 objects detected as dusty candidates in a $D_{\mathrm{Mg} 2}-D_{V K}$ diagram, 11 only have been confirmed by IRAS.

We have produced two-component models with BC03 SSP, with or without dust, for the $3 \mathrm{Pec}$ galaxies quoted above and for 5 reddish objects of the Nop subsample, i.e. NGC 821, $1052,1453,3379$ and 4339, the Wal92 models again being used to quantify the various colour excess. The results are given in Table 6 , both the dusty and dustless model being listed. The following points may be noted:

- ages are given for the SSP component of the models, but these are not really significant;

- in most of the cases the dusty models are formally better, from the values of the $\sigma_{\mathrm{O}-\mathrm{C}}$ test. This is not the case however for NGC 2974, an IRAS bright galaxy, although it appears verified for NGC 3379, an object with zero IRAS flux;

- for the dustless models, the $q_{M}$ percentage of high $Z$ material injected in the postulated SSP "event" is often unrealistic. This parameter is strongly reduced when dust produces part of the reddening of differential colours, but remains rather large. 
Table 6. SSP models of population anomalies: reddish objects in the Pec and Nop subsamples. Same disposition as Table 4. For all objects we give both a dustless model with reddening due only to metal-reach stars $(Z=0.05)$ and a dusty one with reddening due in part to dust. NGC 3923 is apparently dustless.

\begin{tabular}{lllllllllllll}
\hline \hline (1) & $(2)$ & $(3)$ & $(4)$ & $(5)$ & $(6)$ & $(7)$ & $(8)$ & $(9)$ & $(10)$ & $(11)$ & $(12)$ & $(13)$ \\
Name & $\log \sigma_{0}$ & $\Sigma_{2}$ & adip & $f I R$ & $\tau_{V}$ & $A$ & $Z$ & $q_{V}$ & $q_{M}$ & Data & aeq & $\sigma_{\mathrm{O}-\mathrm{C}}$ \\
\hline NGC 2974 & 2.32 & 3.6 & 0.019 & 5200 & 0.0 & 5.00 & 0.050 & 0.20 & 14.6 & - & 2 & 0.91 \\
id & id & id & id & id & 0.25 & 5.00 & 0.050 & 0.09 & 6.6 & - & 2 & 0.96 \\
NGC 3923 & 2.38 & 10.3 & 0.012 & 0 & 0.0 & 8.00 & 0.05 & 0.08 & 8.6 & - & 2 & 1.01 \\
NGC 5846 & 2.39 & 4.2 & 0.023 & 0 & 0.0 & 8.00 & 0.050 & 0.15 & 16.1 & - & 2 & 1.45 \\
id & id & id & id & id & 0.25 & 8.00 & 0.050 & 0.07 & 7.5 & - & 2 & 1.43 \\
\hline NGC 0821 & 2.32 & 0.0 & 0.026 & 760 & 0.0 & 5.00 & 0.050 & 0.40 & 29.3 & - & 2 & 0.84 \\
id & id & id & id & id & 0.5 & 4.00 & 0.050 & 0.20 & 11.8 & - & 3 & 0.77 \\
NGC 1052 & 2.34 & 0.0 & 0.031 & 1710 & 0.0 & 4.00 & 0.050 & 0.30 & 21.7 & nLi & 1 & 1.82 \\
id & id & id & id & id & 0.75 & 4.00 & 0.050 & 0.16 & 9.3 & nLi & 1 & 1.01 \\
NGC 1453 & 2.46 & 0.0 & 0.028 & 6340 & 0.0 & 4.00 & 0.050 & 0.50 & 34.0 & - & 2 & 1.11 \\
id & id & id & id & id & 0.75 & 4.00 & 0.050 & 0.12 & 6.6 & - & 2 & 0.83 \\
NGC 3379 & 2.34 & 0.0 & 0.021 & 0 & 0.0 & 5.00 & 0.050 & 0.20 & 14.5 & - & 2 & 1.18 \\
id & id & id & id & id & 0.25 & 5.00 & 0.050 & 0.14 & 10.1 & - & 2 & 1.07 \\
NGC 4339 & 2.06 & 0.0 & 0.064 & 200 & 0.0 & 4.00 & 0.050 & 0.55 & 46.1 & - & 2 & 1.19 \\
id & id & id & id & id & 1.0 & 4.00 & 0.050 & 0.27 & 18.2 & - & 2 & 1.12 \\
\hline
\end{tabular}

\section{Discussion and conclusion}

\subsection{Comments on the foundations of the present models}

In their simplest form our population models are the combination of a "primary" population, i.e. the average for the Nop ellipticals as a function of $\log \sigma_{0}$, and a "juvenile" one described by an SSP, as resulting from a minor starburst in a recent merger. Our various assumptions will be here briefly discussed.

- In the merger events under consideration, one of the partners at least is a spiral or irregular, containing various numbers of young stars: these will be accreted and partly mixed in the merger product. While the SSP approximation is perhaps adequate to represent the associated starburst, it may be unsatisfactory to describe the actual mix of newly formed and accreted stars. Since these are necessarily older than the starburst they will tend to increase the age of the derived SSP model and also its fractional luminosity $q_{V}$, leading to systematic errors in model parameters. However, "qiescent" star formation in disks does not lead to large mass proportions of newly formed objets, and these fade very rapidly. From $\mathrm{BC} 03$, the stellar $M / L$ ratio of an SSP increases by a factor of 10 between the onset of stellar burning and $A=300 \mathrm{Myr}$ and another factor of 6 to $A=3$ Gyr. We assume therefore the accreted young star component to remain faint compared to the starburst, and the possible systematic errors to remain small.

- If we accept the hierarchical scenario, E-galaxies are formed by mergers of spirals occuring at various epochs: the old stars population of the remnant cannot differ much from the one of the protagonists. Kauffmann et al. (2000) compare the ages of $\mathrm{E}$ forming mergers to the ages of stars in ellipticals: the latter are indeed much larger. This suggests that the primary population derived for E-galaxies is adequate to describe the old population of protagonists in a merger, whether a pair of spirals, or an S0-Sp or E-Sp pair. This is especially true for a recent merger. On the other hand it is a wonder that the process of E-galaxies building has led to such well constrained populations (Nop objects at least), noticeable for the rather tight correlations of indices with mass or luminodity. It is therefore not surprising that significant fluctuations remain in the populations at a given $\log \sigma_{0}$, as this is indeed found in the Nop sample (see Sect. 2.1.1). Reddish objects probably result from an excess of high $Z$ stars in their population, and blueish objects from a deficit of this component. In Sect. 3.2.1 however, another explanation was adopted, and a number of galaxies in the Nop sample modeled with the injection of a young population in a recent event. This interpretation is not compulsory since the assumed event did not leave the expected morphological signature.

- Two star-forming events might occur in so "rapid" a succession that the young stars from the older would significantly modify the initial population present at the onset of the more recent one: the modeling of this situation with our technique would be biased. We have therefore performed numerical experiments in order to estimate the error on the age of a juvenile population induced by the superposition of a somewhat older one (also an SSP), besides the standard primary. This is a problem with many parameters: 3 parameters for the "true" juvenile population, 3 also for the perturbing older one, plus the $\log \sigma_{0}$ defining the primary. We have diminished this number by selecting only one example for the SSP $Y_{0}$ describing the "last star formation event", i.e. the solution of Table 4 for NGC 5061, fairly typical of 
our sample of the younger events, with $A\left(Y_{0}\right)=0.72 \mathrm{Gyr}$. We have added a series of possible older events $O E$, varying their age and mass: then the composite population was analyzed, like an observed object, to provide its modified age and the corresponding error.

Considering first the case of equal fractional mass of the two events $q_{M}(O E)=q_{M}\left(Y_{0}\right)$ the errors on $A\left(Y_{0}\right)$ induced by $O E$ remain small (smaller than from other causes) if $A(O E)$ is only slightly above $A\left(Y_{0}\right)$ say by $0.1-0.2$ Gyr: this might happen when two star-forming events occur during the same galaxy interaction, as the partners oscillate before finally merging, with a typical period of 0.1 Gyr. Errors are also small if the age difference becomes larger than $2 \mathrm{Gyr}$, the population of $O E$ then approaching the "primary" one. Errors however reach $15-20 \%$ in the intermediate range of the age difference $(1<A(O E)<2 \mathrm{Gyr})$. If the masses $q_{M}(O E)$ are larger, twice $q_{M}\left(Y_{0}\right)$ or more, the run of errors with $A(O E)$ is similar, but they are also larger and eventually reach $40 \%$ : the age of the composite population is then "measured" as 1.02 Gyr instead of 0.72 .

Taking the results of 3.1 and 3.2 together we have found out of 114 elipticals, 29 objects with some evidence of star formation more recent than 3 Gyr. If such star productive events are independent in a given object, the probability of pairs of events within the relevant epoch may be reduced to $6-7 \%$. Among these, large errors in dating the "last star formation event" occur only if the older "intruder" is more massive than the recent one by a factor of 2 or more. We may therefore hope that large errors introduced in our results by the succession of more than one star formation event are not frequent.

\subsection{The Pec galaxies with a juvenile population and the theory of mergers}

Several aspects of the theory of mergers are particularly relevant to the discussion of the present work, specifically Sect. 3.1:

1. As already found in the late 70's, the close encounter of two spirals of 1:1 mass ratio is likely to end in a merger, leading to the formation of an E-type galaxy (see reviews by Barnes \& Hernquist 1992; Barnes 1996; Kennicutt 1996; Scweizer 1996).

2. The same happens with mergers up to the 3:1 mass ratio (Barnes 1996).

3. Mergers at larger mass ratios may change the spiral into an object sharing properties of the $\mathrm{S}$ and $\mathrm{E}$ Hubble types, probably an S0 (Naab \& Burkert 2003; Bournaud et al. 2004, 2005).

4. If a galaxy suffers several minor mergers it may become as an elliptical (Bournaud et al. 2005).

5. A merger involving two spirals is necessarily accompanied by a starburst when part of the ISM collapses towards the center of the main object (Barnes \& Hernquist 1996; Mihos \& Hernquist 1996). Empirical arguments to this effects were found in the CO observations (Dupraz \& Casoli 1991; Combes et al. 1994) or in the study of the
Table 7. Available ISM in some Hubble types and merger pairs. The ISM mass $\left(\mathrm{HI}+\mathrm{H}_{2}\right)$ is expressed in terms of the total mass $M_{\mathrm{dyn}}$, DM included. The ratios of interest for comparisons with the $q_{M}$ values of our models should be $M(\mathrm{H}) / M$ (stars) and therefore larger by the factor $M_{\mathrm{dyn}} / M$ (stsrs). M 1: merger E+Sc, 7:1. M 2: merger S0+Sc, 7:1. M3: merger Sa + Sc, 2:1. M 4: merger Sc + Sc, 2:1.

\begin{tabular}{llllllllll}
\hline \hline $\mathrm{E}$ & $\mathrm{S} 0$ & $\mathrm{Sa}$ & $\mathrm{Sb}$ & $\mathrm{Sc}$ & $\mathrm{Sd}$ & $\mathrm{M} 1$ & $\mathrm{M} 2$ & $\mathrm{M} 3$ & $\mathrm{M} 4$ \\
\hline 0.0 & 0.03 & 0.07 & 0.10 & 0.16 & 0.21 & 0.02 & 0.04 & 0.10 & 0.16 \\
\hline
\end{tabular}

prototypical remnant NGC 7252 (Fritze-v. Alvensleben \& Gerhard 1994; Hibbard \& Mihos 1995).

6. A second starburst is possible when another part of the ISM, initially drifting at large distances falls back to the remnant center.

This summary indicates that a large variety of possible mergers involving different progenitors, $\mathrm{S}, \mathrm{S} 0$ and even $\mathrm{E}$, may produce the morphological peculiarities and the starbursts revealed in the juvenile population. One may ask what kinds of mergers are responsible for the above modeled objects, the Pec galaxies of the NGC 2865 family. Our sample does not contain "obvious" merger remnants, all objects being classified E in the RC3. The morphological pecularities and the bluewards population anomalies are more extreme for recent major mergers of the NGC 7252 style. We now propose a classification of mergers according to the Hubble type of the main object, with rough estimates of the amount of ISM available in each case for induced star formation.

1. Class 2SM: initial major merger, types $S+S$, range of mass ratio $1: 1$ to $3: 1$. The output is a remnant, then Epec, then $E$.

2. Class $2 \mathrm{Sm}$ : initial minor merger, types $\mathrm{S}+\mathrm{S}$, range of mass ratio 4:1 and more. The output is a remnant, then an $S 0$.

3. Class S0S: last merger in sequence, types $\mathrm{SO}+\mathrm{S}$, range of mass ratio 4:1 or more. Output: Epec, then E.

4. Class ES: last merger in sequence, types $E+S$, range of mass ratio 4:1 or more. Output: Epec, then E. Here a minor spiral is the "victim" of a large E.

From indications in Young \& Scoville (1991) and Roberts \& Haynes (1994) we have obtained the available ISM mass $\mathrm{HI}+\mathrm{H}_{2}$ in terms of the total dynamical mass $M_{\text {dyn }}$ for the main Hubble types (Table 7). The available ISM in a merger is readily found, given the types of the components and their mass ratio. Exemples are given in Table 7 . To get the $q_{M}$ value of a starburst associated with a merger, one should multiply the available mass by two unknown factors: the ratio of dynamical to stellar mass $M_{\text {dyn }} / M$ (stars) and the efficiency $k_{\text {st }}$ of the starburst.

With these difficulties in mind, our models for Pec galaxies with a juvenile population (Table 4) are tentatively distributed in the above classification on the basis of the massratios $q_{M}$. Candidates for class $2 \mathrm{SM}$ are the objects with the largest $q_{M}$ : NGC 1653, 2865, 4374, 4406, 5576. We cannot believe however, that such giant ellipticals as NGC 4374 and 4406 in the environment of the Virgo Cluster suffered only one merger since their formation. As regards NGC 1653 the 
hypothesis of a major merger is uncertain; the best cases for this classificaion of events are NGC 2865 and NGC 5576. No SO is present in our sample, hence probably no $2 \mathrm{Sm}$ event; NGC 3585 however reaches a diskyness nearly adequate for an S0 and a merger of this class. In the classes SOS and ES we find objects with small $q_{M}$, i.e. NGC 596, 1549, 3557, 3585, $3610,5018,5061,5322,5557,5982$, IC 3370. With an intermediate $q_{M}=4.1 \%$, NGC 4125 and 4976 are of doubtful classification. The model of NGC 1700 contains a young SSP with the smallest $q_{M}$ value observed. It shows rather modest morphological peculiarities (see Paper I; or Schweizer 1996). We consider it as the best candidate for a delayed starburst associated with the ISM falling back to the remant center at the end of the intercation. The referee pointed out to us that the major merger of two early-type objects with little ISM available would produce a remnant with a faint SSP component: in view of the scarcity of S0 as compared to spirals, such events should be quite infrequent.

In merger simulations, the appearance of the remnant is very dependent on both time and the mass-ratios of the protagonists. The timing of the starburst inside the merger event has not been much studied, notably for minor mergers (Class $2 \mathrm{Sm}$ and SOS above). The ages of mergers derived in Sect. 3.1 for the morphologically peculiar galaxies ( $P e c$ sample, $Y P$ family) are in line with the estimates of features durations gathered from the literature, but no detailed comparison seems possible.

\subsection{Comments on other brands of ellipticals}

A number of blueish galaxies with "normal" morphology (Nop subsample) have been modeled (Table 5). If the event described by the calculated SSP did really occur, although we have no morphological evidence, it is possible to attempt the same classification as above: major merger (class 2SM) or last minor merger in a sequence (classes SOS or ES. From their large $q_{M}$ parameters, NGC 4168 and 4478 are the best candidates for the former case, NGC 1600, 2434, 3087, 3250, 3377 for the latter; NGC 584 is doubtful.

For the reddish galaxies of the Pec and Nop sample (Table 6) we cannot determine the age of the event bringing the high $Z$ stars (in the intervall $4-10 \mathrm{Gyr}$ ). The mass fraction $q_{M}$ of these stars is also very uncertain due to the presnce of dust with large effects on colours and therefore on the solution. Even for our dusty models, the $q_{M}$ values are more than $6 \%, 8.6 \%$ for NGC 3923 (no dust) and up to $18.2 \%$ for NGC 4339 (plenty of dust). Such a large mass of high $Z$ stars could be gathered in a single major merger some $5 \mathrm{Gyr}$ ago, if the protagonists had more than average high $Z$ ISM. Perhaps more likely, the reddening of these galaxies is the cumulated result of several star formation episodes, none being recent.

\subsection{Summary of results}

\subsubsection{Technical aspects}

- We derive two-component models of the stellar mix of ellipticals, one being an old "primary" population and the second a "juvenile" SSP, usually from BC03 tables.
Those by W94 are adopted for partial tests. We make use of 10 population indices in well-observed objects, 7 when the Lick indices are missing, to derive 3 parameters (usually), the age $A$, metallicity $Z$ and mass ratio $q_{M}$ of the SSP. This situation is to be prefered to those where the number of observables is equal to (or smaller than!) the number of free parameters to be derived.

- Our models primarily apply to morphologically peculiar galaxies with evidence for the acquisition of young stars, i.e. the Pec subsample and YP subtype (or NGC 2865 family) in the terminology of Paper I. In this case the peculiarities and the presence of the juvenile population probably result from the same event. Models are obtained for $20 \mathrm{such}$ objects (Table 4).

- The effect of diffuse dust can be incorporated in the models, with significant improvement of the results in some cases. The calculations in Wa192 are used for this purpose.

- The differences in young added star content between the central region observed for line-indices and the $r_{\mathrm{e}}$ domain of colour observations, can be taken into account in wellobserved objects with an important young component: only NGC 2865 and 5018 were so treated in this paper. This source of uncertainties could obviously be avoided in specific observations.

- The same kind of modeling may be readily applied to other blueish E-type galaxies in the Nop subsample (without peculiarities), but in this case the results are not supported by the morphological evidence.

- For reddish objects, both in the Pec and Nop subsamples, the technique does not lead to satisfactory models: one needs to introduce large amounts of high $Z$ stars and/or of diffuse dust (Table 6). The age of the SSP component is indeterminate in the 4-10 Gyr range, and its fractional mass $q_{M}$ very uncertain.

\subsubsection{Results}

- In objects of the NGC 2865 family (Table 4), the ages of last star acquisition can be roughly sorted into "young", i.e. $A \leq 1$. and "old", i.e. $A \geq 2$. Young events are associated with relatively strong morphological perturbations, while old events concern objects with more modest peculiarities of the outer isophotes, a rule suffering some exceptions, since parameters other than age, notably the mass ratio, influence the morphology.

- In recent events the $Z$ of the added stars is usually solar, this being an a priori choice, since the $Z$ values at small $A$ are almost indeterminate. Exceptionally a super-solar $Z$ is indicated. In older events the "best" $Z$ are often mildly sub-solar.

- There is an uncertain trend for modeled Pec galaxies to be Fe-rich (and/or Mg-poor), as compared to the standard defined by the $\log \sigma_{0}$ regressions for the Nop (non-peculiar) sample.

- It seems necessary to introduce the effect of diffuse dust in the modeling of a few objects, notably if suggested by 
far IR data (Table 4). The amont of dust is important in IC 3370, the brightest of our sample in the IRAS data.

- In 14 of the 20 modeled objects in Table 4, the mass ratio of the juvenile component is smaller than $3 \%$. This suggests that the responsible merger was not a major one directly leading to the formation of an E-type object, but rather a minor merger bringing the "final touch" to the build-up of the elliptical (Bournaud et al. 2005). This may be also the case for some of the other sample galaxies with larger estimated $q_{M}$.

- Blueish galaxies of the Nop sample can be successfully modeled like the blue objects of the Pec sample. The results are similar, except that the proportion of "old" intruders $(A \geq 2$.) may be higher, while only one object with a "recent" event was found (NGC 3377 with $A=0.81 \mathrm{Gyr}$ ).

- Reddish galaxies of both the Pec subsample (NGC 3923 family) and the Nop subsample can be modeled with the inclusion of both diffuse dust and rather large amounts of high $Z$ stars of age $4<A<10$ Gyr. It is not clear how a single merger could provide such a large quantity of high $Z$ material as needed to form more than $10 \%$ of new stars in a preexisting galaxy. The extra-reddening, i.e. metallicity, was likely built progressively in the past history of such objects, and not by a single event that might be associated with their morphological peculiarities.

Acknowledgements. We thank Drs. F. Combes and F. Bournaud for useful advices about the relations of this work to the theory of mergers, and an anonymous referee for a number of criticisms and suggestions.

\section{References}

Barnes, J. E. 1992, ApJ, 393, 484

Barnes, J. E. 1996 in Galaxies: Interactions and induced Star Formation, Saas-Fe Adv. Course 26 (Springer Verlag), 1998

Barnes, J. E., \& Hernquist, L. 1992, ARA\&A, 30, 705

Barnes, J. E., \& Hernquist, L. 1996, ApJ, 471, 115

Bernlöhr, K. 1992, A\&A, 263, 54

Bernlöhr, K. 1993a, A\&A, 268, 25
Bernlöhr, K. 1993b, A\&A, 270, 20

Bressan, A., Chiosi, C., \& Tantalo, R. 1996, A\&A, 311, 425

Bruzual, G., \& Charlot, S. 2003, MNRAS, 344, 1000 (BC03)

Bournaud, F., Combes, F., \& Jog, C. J. 2004, A\&A, 418, L27

Bournaud, F., Jog, C. J., \& Combes, F. 2005, A\&A, 437, 69

Combes, F., Prugniel, P., Rampazzo, R., et al. 1994, A\&A, 281, 725

Dupraz, C., \& Casoli, F. 1991, in Dynamics of Galaxies and their molecular Clouds Distribution, ed. F. Combes, \& F. Casoli (Dordrecht: Kluwer Acad. Publ.), IAU Symp., 146, 373

Fritze-v. Alvensleben, U., \& Gerhard, O. E. 1994, A\&A, 285, 775

Hibbard, J. E., \& Mihos, J. C. 1995, AJ, 110, 140

Kauffmann, G., Charlot, S., \& Haenelt, M. 2000, Phil. Trans. R. Soc. London Ser. A, 358, 2121

Kennicutt, R. C. Jr. 1996, Galaxies: Interactions and induced Star Formation, Saas-Fe Adv. Course 26 (Springer Verlag), 1998

Knapp, G. R., Guathakurta, P., Kim, D. W., \& Jura, M. 1989, ApJS, 70, 329

Kuntschner, H., Lucey, J. R., Smith, R. J., et al. 2001, MNRAS, 323, 615

Leonardi, A. J., \& Rose, J. A. 1996, AJ, 111, 182

Michard, R. 2005, A\&A, 429, 819 (Paper II)

Michard, R., \& Prugniel, P. 2004, A\&A, 423, 833 (Paper I)

Mihos, J. C., \& Hernquist, L. 1996, ApJ, 464, 641

Naab, T., \& Burkert, A. 2003, ApJ, 597, 893

Roberts, M. S., \& Haynes, M. P. 1994, ARA\&A, 32, 115

Schweizer, F. 1996, in Galaxies: Interactions and induced Star Formation, Saas-Fe Adv. Course 26 (Springer Verlag), 1998

Schweizer, F., \& Seitzer, P. 1992, AJ, 104, 1039

Schweizer, F., Seitzer, P., Faber, S. M., et al. 1990, ApJ, 364, L33

Tantalo, R., \& Chiosi, C. 2002, A\&A, 388, 396

Tantalo, R., Chiosi, C., Bressan, A., et al. 1996, A\&A, 311, 361

Tantalo, R., Chiosi, C., \& Bressan, A. 1998, A\&A, 333, 419

Thomas, D., Maraston, C., \& Bender, R. 2002, Ap\&SS, 281, 371

Thomas, D., Maraston, C., Bender, R., et al. 2005, ApJ, 621, 673

Trager, S. C., Faber, S. M., Worthey, G., et al. 2000a, AJ, 119, 1645

Trager, S. C., Faber, S. M., Worthey, G., et al. 2000b, AJ, 120, 165

Witt, A., Thronson, H. A. Jr., \& Capuano, J. M. 1992, ApJ, 393, 611 (Wal92)

Worthey, G., Faber, S. M., \& Gonzalez, J. J. 1992, ApJ, 398, 69

Worthey, G. 1994, ApJS, 95, 107 (W94)

Young, J. S., \& Scoville, N. Z. 1991, ARA\&A, 29, 581 